\title{
Gobierno electrónico y transparencia financiera y presupuestal de los departamentos en Colombia
}

\author{
Gómez Villegas, Mauricio* \\ Montesinos Julve, Vicente**
}

\section{Resumen}

El gobierno electrónico viene siendo promovido como un instrumento de modernización, que mejora la transparencia y la divulgación de la información financiera y presupuestal de las entidades públicas. En la última década, el e-government ha sido implementado en las entidades gubernamentales latinoamericanas del orden nacional y territorial, por lo que conviene evaluar su grado de avance. El objetivo de este trabajo es evaluar el nivel de divulgación de la información financiera y presupuestal de la administración territorial en Colombia (los departamentos) por medio de Internet, así como identificar las variables que influyen en tales niveles de divulgación. Para ello se utiliza el índice planteado por Caba et al., (2008), ajustado al contexto colombiano. Se miden los niveles de transparencia de 33 departamentos y se identifican, por medio de regresiones, las variables financieras y socioeconómicas que explican los niveles observados. Los resultados muestran que el nivel de divulgación de los departamentos es bajo comparado con la información dispuesta en Internet por el gobierno nacional. Se ha encontrado que el tamaño de la población, el acceso a Internet, el apalancamiento y el estrés financiero, se relacionan significativamente con la divulgación por parte de los departamentos.

Palabras clave: Gobierno electrónico, transparencia de la información, información financiera y presupuestal, Colombia.

\section{Recibido: 16-11-12 Aceptado: 10-03-14}

* Doctor en Contabilidad, Universitat de València. Profesor, Escuela de Administración y Contaduría-Universidad Nacional de Colombia. E-mail: mgomezv@unal.edu.co

** Doctor en Ciencias Económicas, Universitat de València. Catedrático de Economía Financiera y Contabilidad, Departamento de Contabilidad-Universitat de València. E-mail: vicente. montesinos@uv.es 


\section{Electronic Government and Financial and Budgetary Transparency for Colombian Departments}

Electronic government is being promoted as an instrument for modernization that improves the transparency and divulgation of financial and budgetary information by public entities. In the last decade, e-government has been implemented in national and territorial Latin American governmental entities; therefore, their degree of progress should be evaluated. The objective of this work is to assess the level of divulgation for financial and budgetary information from territorial administrations in Colombia (the Departments) through the Internet, as well as to identify the variables that influence these levels of divulgation. To accomplish this, the index proposed by Caba et al., (2008) is used, adjusted to the Colombian context. Transparency levels for 33 departments are measured and, using regressions, the financial and socioeconomic variables that explain the observed levels are identified. Results show that the level of divulgation in the departments is low compared to the information placed on the Internet by the national government. It was also found that the size of the population, access to the Internet, financial leverage and stress are related significantly to divulgation by the departments.

Keywords: Electronic government, transparency of information, financial and budgetary information, Colombia.

\section{Introducción}

Desde principios del siglo XXI surgió un frente de modernización del sector público impulsado por las tecnologías de la información y las comunicaciones (TIC's). Con el apoyo de las TIC's se ha buscado reformar algunos de los procesos de la gestión pública y vincular más activamente a los ciudadanos con la administración. Se pretende que Internet se convierta en una herramienta para disminuir la carga burocrática en los trámites, promover la participación ciudadana, fortalecer la confianza en el Estado y viabilizar e incrementar la transparencia y la rendición de cuentas. Como consecuencia, el gobierno electrónico o e-government emerge y se consolida como un proyecto que impulsa la utilización de In- ternet al servicio de un mejor gobierno (Lapsley y Miller, 2010).

El uso del gobierno electrónico para incrementar la transparencia y la rendición de cuentas ha sido impulsado por instituciones multilaterales, organizaciones no gubernamentales y se viene asimilando en la dinámica de los Estados (Organization for Economic Co-operation and Development-OCDE-, 2003; United Nations -UN-, 2010). Así, la transparencia, la rendición de cuentas y el gobierno electrónico, se incorporan en las expectativas sociales y en las exigencias de los ciudadanos a los gestores públicos.

La Divulgación de Información Financiera y presupuestal del sector público por medio de Internet-DIFI-, como una de las dimensiones del gobierno electrónico, viene siendo objeto de inves- 
tigación académica (La Porte, Demchak y Jong, 2002; Serrano, et al., 2009; Pina, et al., 2010a, 2010b; D'Agostino, et al., 2011; entre otros).

En el contexto internacional, diferentes investigaciones han permitido conocer y comparar los niveles de DIFI (altos, medios o bajos) en los países de la Organisation for Economic Co-operation and Development (OECD). También abundan trabajos que estudian la transparencia en los gobiernos locales de Australia, Estados Unidos y los países de la Unión Europea (Laswad et al., 2005; Caba et al., 2005; Caba et al., 2008; Gandía y Archidona, 2008). La investigación sobre esta materia en la región latinoamericana ha venido en aumento (especialmente en México, Argentina, Chile y Brasil), pero aún requiere mayor exploración empírica (Items y Moreno, 2007; Sour, 2007; Vargas, 2010; Tamez, 2010).

Pese a estos avances en materia institucional y académica, las recientes crisis financiera y de deuda pública en Europa, evidencian malas prácticas y opacidad en la información de la administración pública (International Federation of Accountants, IFAC, 2012). En el contexto latinoamericano, escándalos surgidos en los últimos años comprometen a la administración pública con problemas de corrupción ${ }^{1}$. Estos sucesos cuestionan los resultados alcanzados con las reformas emprendidas para incrementar la transparencia y la divulgación de las cuentas públicas.

En Colombia, la estrategia de Gobierno en Línea cuenta con más de diez años desde su inicio, abarcando los diferentes niveles de la administración públi$\mathrm{ca}$ (local o municipal, regional y nacional). También, en los últimos años, las reformas de la contabilidad gubernamental se han dirigido a potenciar, entre otros aspectos, la rendición de cuentas y la transparencia. En este marco de cosas se hace necesario evaluar los logros de las iniciativas públicas en materia de rendición de cuentas y transparencia por lo que resulta procedente abordar el estudio de la DIFI en Colombia. Con este propósito en este trabajo se han seleccionado a los departamentos, dado que son la instancia de descentralización administrativa y financiera territorial más significativa en el país y debido a que hay fuertes reclamos para mejorar su transparencia y la implementación del e-government (Rodríguez, 2006; Transparencia por Colombia, 2009).

El objetivo de este trabajo es aportar evidencia empírica sobre el nivel de divulgación de la información financiera y presupuestal de la administración pública territorial en Colombia por medio del Internet, así como identificar las variables que influyen en tales niveles de divulgación. Por ello se plantean como preguntas de investigación: ¿Cuáles son los niveles de divulgación de

1 En Latinoamérica los casos de corrupción que comprometen a altos funcionarios de Brasil, Argentina y Colombia, entre otros, han saltado a las principales páginas de los diarios en los últimos cuatro años. 
la información financiera y presupuestal en Internet por parte de los departamentos colombianos? y ¿Cuáles son las variables económicas, financieras y sociodemográficas que explican la divulgación en Internet de la información financiera y presupuestal de los departamentos.

Para responder a estas preguntas se ha seleccionado el índice de DIFI desarrollado por Caba et al., (2008). Este instrumento se compone a su vez de tres índices específicos. Con esta herramienta de medición se determinó el nivel de DIFI de las treinta y tres entidades del gobierno territorial-departamental en Colombia (32 departamentos y el distrito capital). Para explicar los niveles de divulgación observados, se realizan cuatro modelos de regresión, asociados a cada uno de los índices (el general y los específicos). Como variables explicativas se utilizan el acceso a Internet en el departamento, el tamaño poblacional, el ingreso y la riqueza departamental, el nivel de endeudamiento, la magnitud de la inversión y el estrés financiero.

\section{Gobierno electrónico y divulgación de la información pública en Internet}

Inicialmente se presenta una síntesis de la revisión de la literatura asociada al gobierno electrónico, la transparencia y la divulgación de información financiera y presupuestal pública en Internet.

\subsection{Gobierno electrónico}

El New Public Management-NPMtraslada las concepciones, herramientas e instrumentos de la gestión empresarial al sector público, buscando su moderniza- ción (Hood, 1995; Culebro, 2008; Lapsley, 2009). Las TIC's han sido un motor de transformaciones en las empresas y los negocios, permitiendo la instauración de modelos de "negocio a cliente", "negocio a negocio" y "cliente a cliente". Esto ha modificado las relaciones entre las empresas y sus clientes, potenciando la búsqueda de la calidad y de nuevos mercados (Anderson y Zahaf, 2007; Pina et al., 2010b).

La aparente flexibilidad en las transacciones y el fortalecimiento de los vínculos con los clientes, ha llevado a plantear a los más entusiastas seguidores del NPM, que el traslado de los modelos de "negocio a negocio" y "cliente a cliente" al sector público, permitirán construir una democracia electrónica (D'Agostino et al., 2011). En este contexto surge el gobierno electrónico o e-government (Lapsley y Miller, 2010; Pina et al., 2010b), definido como "el uso de las tecnologías de la información y las comunicaciones, y particularmente de Internet, como una herramienta para alcanzarun mejor gobierno"(OECD, 2003; 11).

La literatura académica caracteriza cinco etapas en la evolución del gobierno electrónico (Moon, 2002; OECD, 2003; National Audit Office, 2002; Pina et al., 2010a). En la primera se utiliza el Internet como un medio para difundir información genérica y descriptiva de la administración pública. La segunda se distingue por la prestación de algunos de los servicios públicos tradicionales en la plataforma web y por fortalecer la provisión de información sobre los logros de la gestión pública. Por su parte, la tercera se consigue el tránsito a un portal transaccional, que dispone en Internet la gran mayoría de trámites para los ciudadanos (Pina et al., 2010a). 
Las dos etapas restantes suponen una transformación de la gestión y la burocracia pública, consiguiendo una vinculación sustancial del ciudadano y alcanzando una democracia electrónica. Por consiguiente, en la cuarta se busca una web integrada a los procesos administrativos de la gestión pública como un todo. La quinta sería el logro de la democracia interactiva, en la que los ciudadanos participan directamente (en línea) en las decisiones y el control de la administración pública, alcanzando mayores grados de responsabilidad y transparencia (Moon, 2002; Pina et al., 2010a). Hay evidencia de la implementación de las tres primeras etapas en países avanzados, emergentes y en vías de desarrollo. Menos clara es la materialización de las etapas cuatro y cinco (Moon, 2002; King, 2006; UN, 2010).

En Latinoamérica, las investigaciones revelan resultados similares a los observados en los países de la OCDE, mostrando la dificultad de llegar al cambio organizacional (Sour, 2007; Vargas, 2010). Variables contextuales tales como la tradición legal, la cultura política y los modelos de administración pública, implican matices en la explicación de los obstáculos y avances conseguidos en Latinoamérica (Rodríguez, et al., 2006; Vargas, 2010). Por ejemplo, un impulso significativo del gobierno electrónico en los países con tradición de derecho latino, ha sido la emisión de leyes para incorporar las nuevas tecnologías ${ }^{2}$, antes que las demandas directas de la ciudadanía.

En Colombia, desde el año 2000, "Gobierno en línea ${ }^{3}$ se convierte en el mejor instrumento para fomentar la competitividad, el crecimiento económico, el Buen Gobierno y en general propicia un ambiente para la innovación Gubernamental que lleve a la prosperidad del país" (Ministerio de las Tecnologías de la Información y las Comunicaciones -MTIC-, 2011; 11). La estrategia de Gobierno en línea establece cinco (5) fases para conseguir una mejor gestión pública con el apoyo del e-government. Según la estrategia, en este momento la mayoría de entidades públicas deberían haber superado la fase tres o de "transacción" (MTIC, 2011).

El ranking de las Naciones Unidas sobre gobierno electrónico ubica a Colombia en el lugar 31 para el año 2010 y en el puesto 43 en 2012, lo que muestra su aparente liderazgo en la implementación del e-government en la región (United Nations, 2010, 2012). No obstante, las investigaciones académicas señalan que el avance del gobierno electrónico en el país no es tan relevante, requiriendo transformaciones culturales y organizacionales en el Estado, el sector empresarial y la ciudadanía. Se manifiesta escepticismo y una brecha significativa en la implantación de Gobierno en Línea entre las

2 En Colombia, la Ley 962/2005 y el decreto 1151/2008 estructuran las bases de gobierno en línea, estrategia de gobierno electrónico del Estado colombiano.

3 Para mayor información puede consultarse el sitio web: http://www.gobiernoenlinea.gov.co/web/guest_ 
entidades del orden nacional y las del territorial (Rodríguez, 2006).

Desde un punto de vista crítico, la evaluación internacional de los logros en la implantación del gobierno electrónico está revelando las complejas relaciones existentes entre consultores, empresas de ingeniería e informática, políticos y funcionarios públicos (Lapsley, 2009). A los tradicionales obstáculos para el cambio organizacional que conllevan al fracaso de diferentes reformas en el sector público, en el caso del gobierno electrónico hay que adicionar los intereses que surgen del lucrativo mercado de consultoría y asesoría en sistemas (Christensen y Skaerbaek, 2010). También hay serias críticas por la asimilación de los ciudadanos con clientes y las implicaciones de este tratamiento.

\subsection{Transparencia}

El gobierno electrónico pretende impactar en la transparencia, puesto que supone mejoras en la información divulgada por la administración pública. "Transparencia, en términos generales, significa conducir las actividades de una manera en que las decisiones, las reglas y otra información se hacen visibles desde el exterior" (Hood, 2010; 989). No obstante, esta definición genérica de transparencia es criticada porque podría promover la retórica. Se argumenta que en la sociedad contemporánea ningún agente se muestra contario, a priori, a la transparencia (Hood, 2010).

Por lo previamente señalado, ante la posibilidad del uso retórico del concepto, conviene precisarlo para hacerlo operativo y contrastable. Así, para el objetivo de este trabajo se asume que: "...la transparencia en la sociedad de nuestros días significa información fácilmente accesible, fiable, que sea relevante para las decisiones, que resulte comparable y que se presente de forma clara y oportuna. En definitiva, que cumpla los requisitos y características cualitativas del marco conceptual de la contabilidad, con un especial énfasis en la accesibilidad, derivado del papel revolucionario del Internet" (Montesinos, 2009: 26).

El estudio de la transparencia de la administración pública en Internet y de la expansión del gobierno electrónico, ha configurado un área de investigación académica vinculada con la gestión y la contabilidad pública. El diseño y aplicación de diversos índices de divulgación ha sido un frente de trabajo creciente $(\mathrm{Ho}$, 2002; Moon, 2002; La Porte et al., 2002; Rodríguez, et al., 2006; West 2004; Laswad, et al., 2005; Caba, et al., 2005, 2008; Gandía y Archidona, 2008; Pina et al., 2010a, 2010b; D'Agostino et al., 2011; entre otros). Con estos instrumentos se mide el grado de divulgación, caracterizando el contenido, la calidad y la accesibilidad a la información contable y presupuestal pública divulgada en Internet.

Con los resultados se realizan comparaciones entre entidades, en el ámbito local, regional e internacional. Las investigaciones también han identificado las variables sociales, demográficas, económicas y políticas que explican un determinado nivel de divulgación, como variable subrogada de la transparencia.

Transparencia por Colombia, oficina de la organización Transparency International, desarrolló desde 2004 y hasta 2009 un índice para medir la transparen- 
cia del sector público, tanto en el gobierno central como descentralizado ${ }^{4}$. El índice de transparencia de los departamentos, recoge tres (3) aspectos: 1 ) visibilidad, 2) institucionalidad y, 3) sanción y control (Transparencia por Colombia, 2009). Estas mediciones no resultan específicas ni suficientemente comprensivas del rol de la divulgación y la transparencia de las cuentas financieras y presupuestales.

\subsection{Divulgación de información financiera y presupuestal pública en Internet (DIFI)}

En general, la DIFI no es obligatoria. Sólo recientemente algunos países han buscado la divulgación obligatoria de alguna información presupuestal en los sitios web de las entidades públicas ${ }^{5}$. Esta tendencia emula las exigencias a las empresas cotizadas que busca reforzar el gobierno corporativo.

Dada la ausencia de regulación u obligatoriedad, las teorías sobre la divulgación voluntaria han sido el referente para el estudio de la DIFI (Laswad et al., 2005; Gandía y Archidona, 2008). Así, son ampliamente utilizadas en la literatura: la teoría de la agencia, la teoría institucional (Collin et al., 2009) y la teoría de la elección pública - Public Choice- (Chan y Rubin, 1987; Ball y Pflugrath, 2012).

Las interacciones entre ciudadanos y políticos (los cargos de elección po- pular que llegan al gobierno) han sido caracterizadas a la luz de la teoría de la agencia (Zimmerman, 1977; Caba et al., 2008; Gandía y Archidona, 2008). Allí se asume que los ciudadanos y los políticos son sujetos racionales que buscan maximizar su beneficio particular y que tienen intereses que pueden entrar en conflicto.

La información divulgada hace parte de los mecanismos e instrumentos de coordinación para disminuir los costos de agencia. La DIFI se explica por el interés de los políticos (los agentes) de ser relegidos. Al mismo tiempo, la divulgación realizada disminuye la asimetría de información para que los ciudadanos (los principales) puedan tomar decisiones y ejercer un control mejorado sobre las acciones de los agentes (Collin et al., 2009; Pina et al., 2010b). La literatura extiende el rol de los principales, también, a otros interesados como los proveedores de crédito al gobierno (Collin et al., 2009).

Desde otro punto de vista, la DIFI puede ser explicada por la teoría institucional (Collin et al., 2009; Pina et al., 2010b). Esta teoría plantea que las organizaciones asumen estructuras, procedimientos y técnicas no necesariamente como un medio para conseguir mayor racionalidad y eficiencia (DiMaggio y Powell, 1983). Las organizaciones tienden a parecerse entre sí, porque existen presiones institucionales en sus respectivos contextos, que las llevan a asumir

Para mayor información puede consultarse el sitio web: http://www.transparenciacolombia.org.col 
comportamientos de isomorfismo (Pilcher, 2011). Establecer una determinada estructura organizacional, incorporar una técnica o una tecnología o implantar estrategias, planes y procedimientos, serían vías de los gerentes para mostrar a la organización como apegada a los valores dominantes o a las expectativas sociales. La DIFI sería entonces explicada como un mecanismo mimético (imitando a organizaciones líderes), como una estrategia normativa (siguiendo las indicaciones de tecnócratas o expertos) o por una presión coercitiva (para responder a normas o directrices de agencias estatales superiores) (Collin et al., 2009).

Por su parte, la teoría de la elección pública señala que los votantes tienen un comportamiento económico racional, llevándolos a tomar decisiones de movilidad hacia las regiones en las cuales sus beneficios se maximizan. Los impuestos, así como las tasas y precios por servicios públi$\cos$, constituyen la variable que los ciudadanos buscan gestionar. Los votantes esperan los mayores logros de la administración pública en términos de servicios, al tiempo que buscan la disminución de su carga fiscal (Chan y Rubin, 1987). La información financiera y presupuestal pública permite hacer seguimiento al uso de los impuestos y de las cargas asumidas por los ciudadanos. Por consiguiente, los políticos en el gobierno divulgan esta información no sólo para el electorado de su jurisdicción, sino para aquellos ciudadanos residentes en las comunidades vecinas y que son electores potenciales. De esta manera, la divulgación de información es un medio para mantener, y promover el aumento de, votos que garanticen la relección del político en el gobierno (Chan y Rubin, 1987; Ball y Pflugrath, 2012).
La evidencia en la investigación internacional muestra que los estilos de gestión (Pina et al., 2010a, 2010b) la tradición del sistema legal y administrativo (Rodríguez et al., 2006) y las condiciones contextuales culturales (D'Agostino et al., 2011) tienen un impacto significativo en el nivel de divulgación. Aplicando análisis de cluster, se identifica que los países de tradición anglosajona, muestran mejores índices de divulgación, en general, que los países con estilos administrativos europeos del sur, alemán e incluso nórdicos (Pina et al., 2010a; 2010b).

La literatura previa identifica algunas de las variables demográficas, sociales, políticas y económicas que tienen una influencia significativa en el nivel de DIFI. El tamaño poblacional, la riqueza del municipio, el porcentaje de acceso a Internet en los hogares, el nivel educativo de los ciudadanos, la visibilidad del gobernante en los medios de comunicación, el grado de endeudamiento, el partido de gobierno, el volumen de ingresos, la presión fiscal, el volumen del déficit o del superávit, el costo del endeudamiento, entre otras, han sido variables ampliamente contrastadas en la investigación sobre la DIFI (Laswad et al., 2005; Caba et al., 2008; Gandía y Archidona, 2008; Pina et al., 2010b; D'Agostino et al., 2011; entre otros).

En el caso Colombiano, no existe un antecedente de medición de la divulgación de información contable y presupuestal de los departamentos. Un esfuerzo desactualizado es el índice de transparencia por Colombia, el cual, en su componente de "visibilidad", recoge algunos elementos de divulgación en Internet de información general presupuestal (Transparencia por Colombia, 2009). 


\section{Evidencia empírica de la DIFI en los departamentos de Colombia}

En este apartado se presenta el índice DIFI seleccionado, el diseño metodológico adoptado y se exponen los resultados descriptivos y explicativos de la divulgación de información financiera y presupuestal en internet de los departamentos en Colombia. En la parte final se realiza una discusión de los hallazgos.

\section{1. Índice adoptado y población de estudio}

En ausencia de regulación para la divulgación en Internet es usual tomar como referente para la construcción de índices, algunos ítems de la información obligatoria que deben presentar las entidades públicas a las instancias de control y/o supervisión. Se incorporan también otros criterios asociados a las características cualitativas de la información, a las expectativas de interactividad de los sitios web, entre otros (Asociación Española de Contabilidad y Administración de Empresas, AECA, 2005; Rodríguez et al., 2006; Gandía y Archidona, 2008; Caba et al., 2008; Pina et al., 2010a, 2010b).

Se ha seleccionado el índice que desarrollaron Caba et al., (2008) ya que ha sido utilizado para estudiar la DIFI en gobiernos nacionales y territoriales en Europa y América. El índice general está compuesto por tres grupos de criterios: contenido, características cualitativas y navegabilidad, qué permitirán construir tres índices específicos.

Para reconocer las particularidades del contexto colombiano, algunos de los criterios fueron ajustados. Se modificaron los ítems relacionados con el tipo de informes contables y presupuestales, en el marco del Régimen de Contabilidad Pública -RCP- (Contaduría General de la Nación, 2007) y del Estatuto Orgánico del Presupuesto (Ministerio de Hacienda y Crédito Público, 1996).

Cada uno de los tres grupos de criterios permite la medición de un índice específico, así:

Índice de Contenido de Información - $\mathrm{ICl}-$ : Permite medir la divulgación en Internet de los informes contables y presupuestales que las normas exigen preparar a las entidades contables públicas en Colombia. También, refleja la difusión de indicadores no financieros sobre el desempeño de la gestión de la entidad. El índice específico es la sumatoria de cada puntaje obtenido según los criterios establecidos de contenido de la información (CEc).

Índice de Características Cualitativas -ICC-Este índice refleja el grado en el cual la información divulgada atiende a las características cualitativas de la información contenidas en el marco conceptual de la información financiera ${ }^{6}$ (International Accounting Standards Committee, 1999; Financial Accounting 
Standards Board, 2000; Caba et al, 2008). Se extiende el uso de estas características a la información presupuestal. Este índice da cuenta indirecta de los efectos de la reforma de la gestión financiera pública sobre la calidad de la información y sobre la transparencia, por su alineación con la definición que hemos adoptado de la misma. El índice específico es la agregación de cada puntaje obtenido según los criterios establecidos de características cualitativas (CEci).

Índice de Navegabilidad y Acceso -INA- Por medio de este índice, se determina el grado en que el sitio web oficial promueve el acceso a la información divulgada. El índice específico enfatiza en la navegabilidad, accesibilidad y manejo de la información y datos que de ella se pueden extraer. El índice específico es la sumatoria de cada puntaje obtenido según los criterios establecidos de navegabilidad y acceso $\left(C E_{n}\right)$.

Finalmente, el Índice General de Divulgación -IGD- está constituido por la agregación de los puntajes de cada grupo de criterios, o índices específicos y se puede expresar como:

$$
I G D=\sum_{i=1}^{I} C E_{c}+\sum_{j=1}^{m} C E_{c i}+\sum_{k=1}^{n} C E_{n}
$$

Donde $C E_{c}, C E_{c i}$ y son los valores asignados a cada criterio establecido o ítem incluido para contenido de información, características de la información y navegabilidad y accesibilidad, respectivamente. Cada criterio es punteado según si el departamento lo observa en la información divulgada y/o en su sitio web. Si el criterio no es observado, se puntea con 0. En la Tabla 1 se presentan los ítems recogidos en el índice seleccionado, así como su valoración, siguiendo los criterios y puntajes establecidos por Caba et al., (2008).

En Colombia, los departamentos y el Distrito Capital son las instancias más representativas de la descentralización territorial regional (Asamblea Nacional Constituyente, 1991; Artículo 298). Las Gobernaciones son las entidades de gobierno departamental. En el Distrito Capital, la Alcaldía Mayor es el órgano de gobierno. El acceso al poder político como gobernador o alcalde se da por medio de elección popular (Asamblea Nacional Constituyente, 1991; Artículo 259). En total existen en el país 32 departamentos y un Distrito Capital. Se ha seleccionado esta población para realizar la investigación.

La fecha de corte para contrastar la información contable y presupuestal divulgada es 2010, buscando homogeneidad y comparabilidad, dado que son los datos consolidados y validados más próximos al momento de la investigación. La exploración en los sitios web de las entidades para la construcción de los índices fue ejecutada entre abril y mayo de 2012.

\subsection{Análisis de los índices DIFI}

A continuación se presentan los resultados obtenidos fruto de la exploración y desarrollo de los índices seleccionados. Primero se presentan los hallazgos descriptivos, luego se sintetizan las hipótesis, los modelos y la evidencia explicativa que permiten las regresiones por Mínimos Cuadrados Ordinarios, MCO. 


\section{Tabla 1}

Composición, criterios y puntajes del índice de DIFI seleccionado

\begin{tabular}{lcc}
\hline \multicolumn{1}{c}{ Índice de divulgación y transparencia e-government } & $\begin{array}{c}\text { Puntuación } \\
\text { por ÍTEM }\end{array}$ & $\begin{array}{c}\text { Puntaje } \\
\text { total }\end{array}$ \\
\hline 1. CONTENIDO DE LA INFORMACIÓN PROVISTA & 6 \\
\hline 1.A. Información presupuestal y flujo de caja & 2 \\
1.A.1. Información del presupuesto del año fiscal & 0,40 \\
1.A.2. Gastos ejecutados & 0,40 \\
1.A.3. Ingresos ejecutados & 0,40 \\
1.A.4. Resultado presupuestal & 0,40 \\
1.A.5. Modificaciones presupuestales & 0,40 \\
1.B. Posición Financiera & 2 \\
1.B.1. Balance General & 0,40 \\
1.B.2. Estado de Actividad & 0,40 \\
1.B.3. Estados de Cambios en el patrimonio & 0,40 \\
1.B.4. Notas a los Estados Contables & 0,40 \\
1.B.5. Información del Endeudamiento & 0,40 \\
1.C. Información no Financiera & 2 \\
(relacionados con la gestión de la administración pública) & \\
1.C.1. Indicadores de Eficiencia & \\
1.C.2. Indicadores de Economía & 0,67 \\
1.C.3. Indicadores de Eficacia & 0,67 \\
\hline
\end{tabular}

2. CARACTERÍSTICAS DE LA INFORMACIÓN

2.A. Integridad

La página web debe ser tan informativa como sea posible

2.A.1. Las cuentas anuales pueden ser consultadas en la página web 1

2.B. Oportunidad 1

Provee información de manera más regular que simplemente anual

2.B.1. Conveniencia oportunidad de la información provista 1

(mensual-trimestral)

2.C. Comparabilidad

Provee información para tres o más periodos

2.C.1. Existe la posibilidad de hacer comparaciones

2.C.2. Son provistos resúmenes comparativos de la inf. contable.

2.D. Comprensibilidad

Hay gráficos y ratios, con comentarios explicativos

2.D.1. Ratios, gráficos o figuras de respaldo se han introducido a la $\quad 0,5$ información

2.D.2. Son incluidos comentarios a la información contable 0,5 


\section{Tabla 1 (Continuación)}

Índice de divulgación y transparencia e-government

Puntuación Puntaje por ÍTEM total

2.E. Relevancia

1

Provisión de reportes técnicos sobre el desempeño gerencial

2.E.1. Están disponibles reportes técnicos de la propia entidad

2.E.2. La información ofrecida está ordenada y clasificada

0,5

2.F. Confiabilidad

La información es verificada por auditores.

2.F.1. La información Financiera oficial está certificada

2.F.2. La información auditada y no auditada es claramente diferenciada

3. NAVEGABILIDAD, DISEÑO Y ACCESO

3.A. Fácil acceso a la información

Existencia de un mapa del sitio y de una sección sobre inf. Financiera

3.A.1. La página web tiene una sección para inf. Financiera y presupuestal pública

3.A.2. El mapa del sitio muestra la inf. que está disponible

Se establecen diferentes perfiles de acceso a la información

3.B.1. Se establece acceso limitado a ciertas áreas por perfil del usuario

3.C. Fácil desplazamiento a través de las áreas de inf. Financiera

Se establecen hipervínculos dentro de las secciones de inf. financiera

3.C.1. Un sistema de hipervínculos para la inf. es provisto

3.D. Fácil gestión de los datos

La información esta disponible en diferentes formatos para descarga.

3.D.1. Formato: html

3.D.2. Formato: pdf o doc

3.D.3. Formato: xls

3.E Fácil uso en un contexto internacional

Es positivo que la información esté disponible en diferentes idiomas

3.E.1. El contenido de la información está en diferentes idiomas

3.F. Interactividad con el usuario

Medios disponibles para la interactividad entre el usuario y la administración

3.F.1. Hay un e-mail disponible diferente al webmaster, para explicaciones y requerimientos

3.F.2. Una lista de correos electrónicos es provista 


\subsubsection{Resultados descriptivos}

Los estadísticos descriptivos de los Índices de DIFI de los departamentos en Colombia, son presentados en la Tabla 2.

Se observa que la media del Índice de Contenido de la Información es de 1,725. Esto implica que, en promedio, los departamentos divulgan el $28,75 \%$ de la información contable y presupuestal ideal. Este porcentaje es muy bajo comparado con los resultados del mismo índice aplicado a la DIFI de los gobiernos centrales de los países latinoamericanos, donde se alcanza una divulgación promedio del 82,5\% (Rodríguez et al., 2006). Ningún departamento cumple con todos los criterios del contenido de información planteados.

El gráfico 1 presenta datos específicos del contenido de la información. Los informes más divulgados son presupuestales, alcanzando en promedio un $44,24 \%$ de la información ideal. Resaltan los informes de ingresos y gastos ejecu- tados, ya que $57,58 \%$ de los departamentos los disponen en Internet. La divulgación de información contable tiene un menor nivel que la presupuestal. En promedio, solo se dispone en Internet el 27,88\% de la información que es obligatorio elaborar según el RCP. El informe financiero que más se divulga es el Balance General, presentado en forma electrónica por 13 departamentos, lo que representa el $39,39 \%$ de la población. Existen 4 departamentos, el $12,12 \%$, que disponen en Internet para los interesados algún tipo información sobre el endeudamiento de la entidad.

En cuanto a los indicadores de gestión, 3 departamentos colombianos, el $9,09 \%$, presentan algunos de ellos para informar sobre la eficiencia y el $33,33 \%$, es decir 11 , divulgan indicadores sobre eficacia o cumplimiento de metas. Esto se debe, en parte, a la obligatoriedad de realizar audiencias públicas de rendición de cuentas anuales, frente a la ciudadanía, para los cuales se preparan estas mediciones.

\section{Tabla 2}

Estadísticos descriptivos de los índices específicos y general

\begin{tabular}{ccccc}
\hline & ICI & ICC & INA & IDG \\
\hline Valid & 33 & 33 & 33 & 33 \\
$\mathbf{N}$ & & & & \\
Missing & 3 & 3 & 3 & 3 \\
Mean & 1,725258 & 1,742424 & 2,795455 & 6,263136 \\
Median & 1,600000 & 1,00000 & 2,500000 & 4,650000 \\
Std. Deviation & 1,5318651 & 1,8545603 & 1,1800002 & 4,1611518 \\
Minimum &, 0000 &, 0000 & 1,0000 & 1,0000 \\
Maximum & 4,6667 & 5,5000 & 5,2500 & 13,8500 \\
\hline
\end{tabular}

Fuente: Elaboración propia a partir de índices y resultados de exploración en páginas web de los departamentos en 2012. 


\section{Gráfico 1 \\ Contenido de la información contable y presupuestal divulgada por los departamentos colombianos}

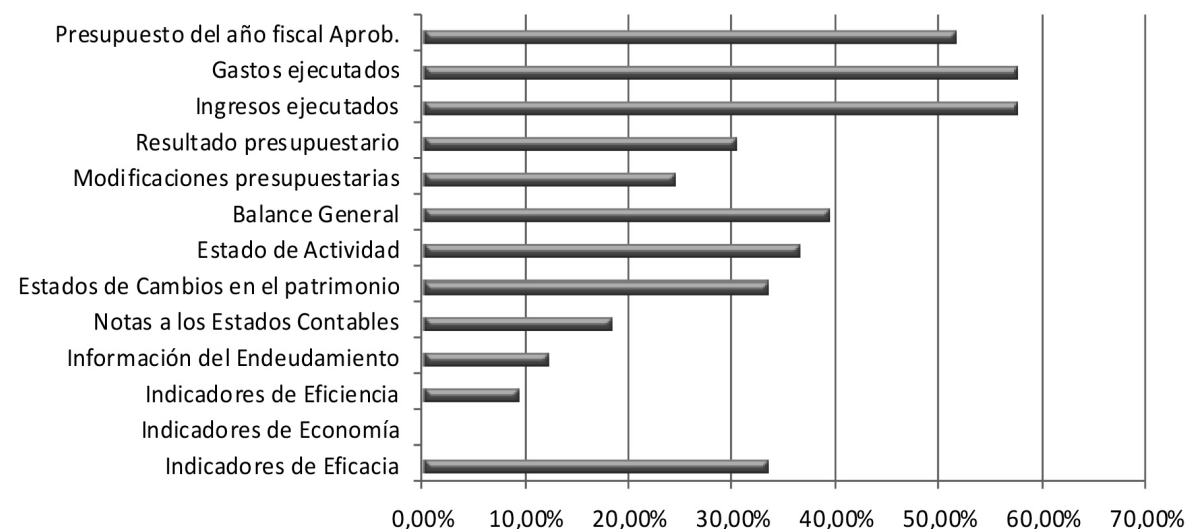

Fuente: Elaboración propia a partir de índices y resultados de exploración en páginas web de los departamentos en 2012.

El Gráfico 2 explicita los criterios relativos al Indicador de Características Cualitativas. Dado el promedio de 1,7424, puede decirse que, en conjunto, la información divulgada por los departamentos sólo cumple con el $29,04 \%$ de las cualidades de la información, según nuestro instrumento de medición. Como muestra el gráfico, una de las características de mayor observancia es la relevancia, ya que 22 departamentos, el $66.67 \%$, divulgan la información atendiendo a criterios de clasificación y orden.

La comprensibilidad es una característica crítica en la información territorial publicada en Internet puesto que sólo 4 departamentos, el $12,12 \%$, complementan los informes con datos, cuadros o gráficos para ayudar a su interpretación y comprensión por parte de los usuarios. Tampoco se garantiza que la información sea percibida como altamente confiable, puesto que en ningún caso en la información divulgada se ex- plicita si es auditada o no. Sólo 12 departamentos, el $36,36 \%$, presentan los informes contables debidamente firmadoscertificados por sus elaboradores y por los gestores responsables.

El gráfico 3, detalla los porcentajes observados para cada criterio que componen el Índice de Navegabilidad y Acceso, INA. Este es el índice que obtiene el mayor promedio, 2,7954. Para ningún departamento este índice tomó el valor 0 . Esto se debe a que todos tienen un sitio web oficial y como mínimo observan uno (1) de los criterios incorporados en el instrumento. El formato más usado para la DIFI es el PDF, que es utilizado por el $75,76 \%$, es decir 25 entidades territoriales. Todos los departamentos disponen y hacen pública una dirección de correo electrónico para atender solicitudes ciudadanas. El acceso al sitio web en un segundo idioma es posible en 15 casos, lo que representa el $45,45 \%$. 


\section{Gráfico 2}

\section{Características de la información contable y presupuestal divulgada por los departamentos colombianos}

Integridad: divulgación de informes completos

Oportunidad: (mensual-trimestral)

Comparabilidad: info de varios periodos

Comparabilidad: resúmenes comparativos de. Comprensibilidad: hay Ratios, gráficos o figuras. Comprensibilidad: Son incluidos comentarios a..

Relevancia: Reportes técnicos de la propia...

Relevancia: inf. ofrecida está ordenada y.

Confiabilidad: La información Financiera esta...

Confiabilidad: La inf. auditada y no audit. es.

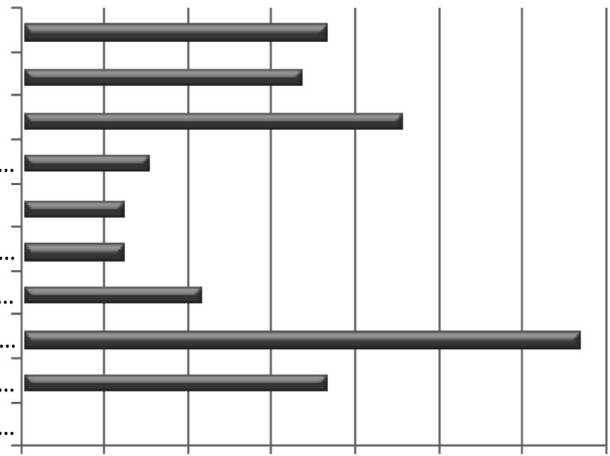

$0,00 \% 10,00 \% 20,00 \% 30,00 \% 40,00 \% 50,00 \% 60,00 \% 70,00 \%$

Fuente: Elaboración propia a partir de índices y resultados de exploración en páginas web de los departamentos en 2012.

\section{Gráfico 3 \\ Navegabilidad y accesibilidad a la información contable y presupuestal divulgada por los departamentos colombianos}

Fácil acceso: sección de inf. finan y presup. Fácil acceso: mapa del sitio muestra la info disp.

Categorización: Difer. Perfiles de usuarios

Navegabilidad: hay hipervínculos para la inf.

Gestión datos: Formato html.

Gestión de datos: Formato pdf o doc.

Gestión de datos: Formato xls.

Perfil internacional: Contenido otros idiomas. Interacción: entrega e-mail para requerimientos Interacción: Una lista de correos electrónicos...

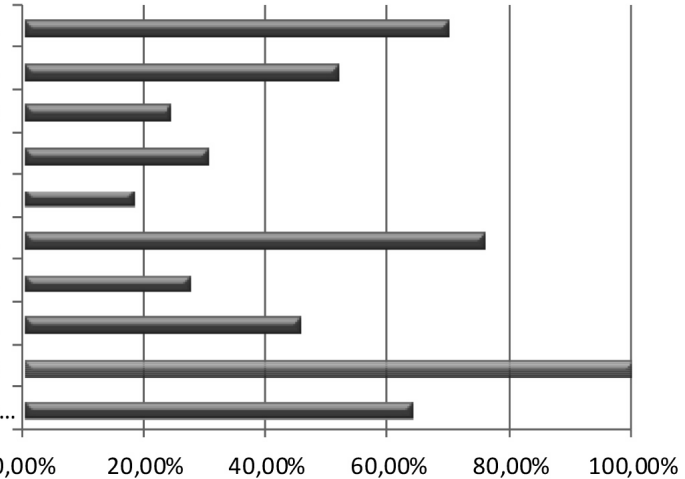

Fuente: Elaboración propia a partir de índices y resultados de exploración en páginas web de los departamentos en 2012.

En cuanto al Índice General de Divulgación, el promedio es de 6,2631. Esto quiere decir que, considerado sobre el puntaje global posible de 18 puntos, las entidades territoriales departamentales en Colombia obtienen un nivel de DIFI de $34,80 \%$. Las Tablas 3 y 4 presentan a continuación la relación de los 10 departamentos con mejores y los 10 con peores índices, respectivamente. 


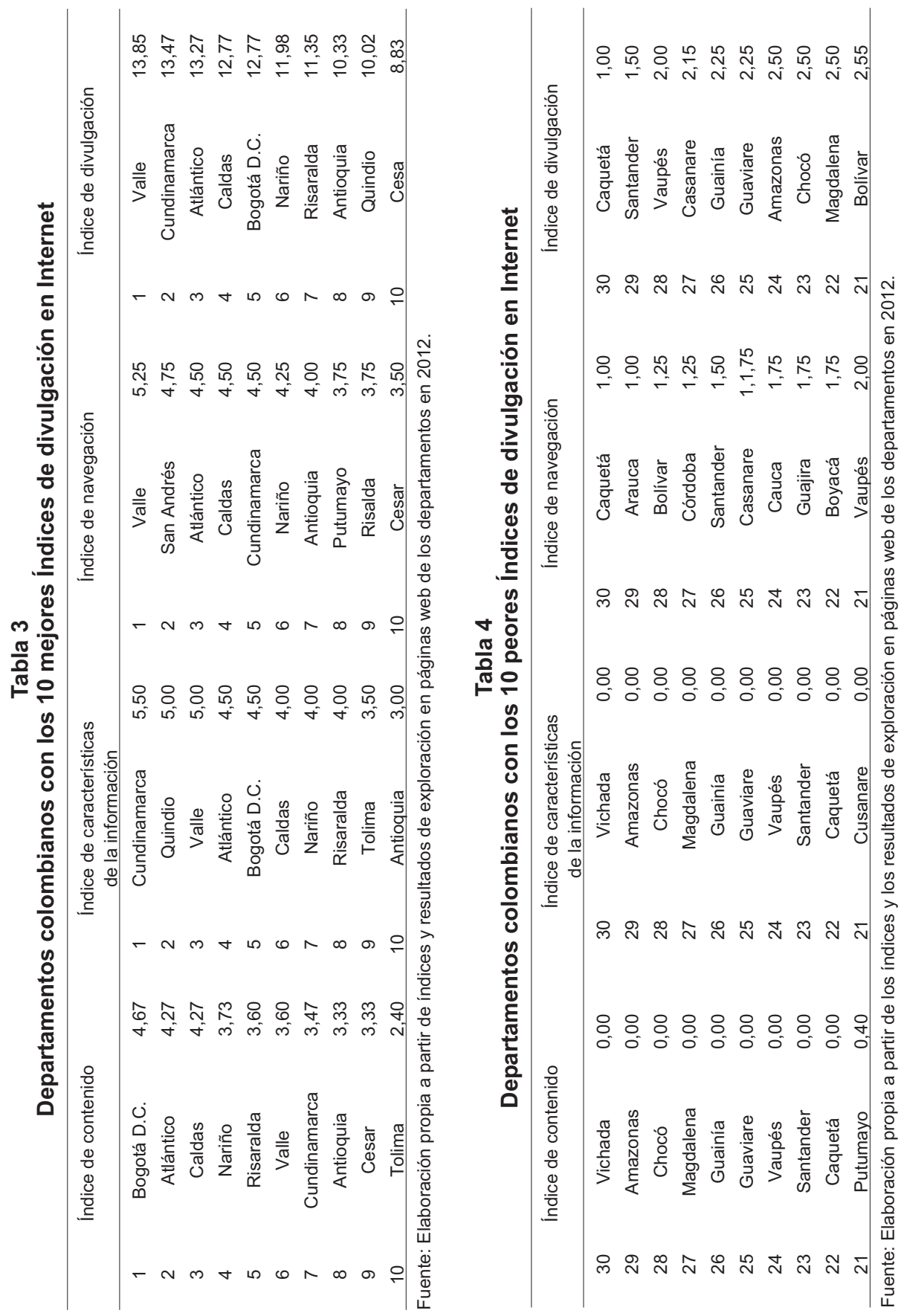


Se destaca el departamento del Valle del Cauca, que ocupa el primer lugar, con un índice de 13,85, lo que significa un grado de divulgación del 76,94\%. En la décima posición se ubica el Departamento del Cesar con un índice de 8,83, lo que es un nivel de DIFI del $49,07 \%$. Esto quiere decir que las primeras nueve posiciones consiguen un nivel de divulgación superior al $50 \%$.

En las peores posiciones se ubican los departamentos que antes de la constitución de 1991 se denominaban "intendencias y comisarías" y que históricamente muestran un rezago significativo en indicadores socioeconómicos. En el rango del nivel de divulgación del 0 al $20 \%$ se ubican un total de 13 departamentos. Esto significa que casi el $40 \%$ de las entidades territoriales tienen niveles de divulgación inferiores al $20 \%$ de lo ideal.

Una circunstancia atípica se evidencia con la divulgación de Santander, pues tradicionalmente este departamento se destaca por su liderazgo en la implantación de Gobierno en Línea (Transparencia por Colombia, 2009). En el momento de la exploración, el sitio web de esta gobernación se encontraba en transformación y no estaba disponible la información financiera y presupuestal. Para evitar una distorsión, luego de una evaluación previa, se eliminó esta observación atípica en los modelos de regresión (Wooldridge, 2006; 344-345).

\subsubsection{Hipótesis y variables que explican la DIFI de los departamentos colombianos}

Para explicar las variables que influyen en la DIFI de los departamentos, son retomados los argumentos de la teo- ría de la agencia y de la teoría de la elección pública.

El acceso a Internet es una variable socio-demográfica que podría promover u obstaculizar la implantación del gobierno electrónico en una región o área geográfica (Debreceny et al., 2002; Caba, et al., 2008; D'Agostino et al, 2011). Las relaciones entre gobierno electrónico, acceso a la tecnología y nivel educativo han sido estudiadas en la literatura previa sobre DIFI (Gandía y Archidona, 2008; Caba et al, 2008). Como consecuencia se plantea que:

H1. Hay una asociación positiva entre el acceso a Internet en el departamento y la divulgación de información financiera y presupuestal en los sitios web de los departamentos colombianos.

En la literatura se plantea que los políticos en el gobierno de las entidades que controlan más ingresos, cuentan con recursos suficientes que les permiten emprender actividades de divulgación de su gestión para promover su reelección. Al mismo tiempo, cuando una entidad territorial dispone de más recursos, los ciudadanos tienen más incentivos para exigir, presionar y buscar acceder a información en los nuevos canales de divulgación (Ingram, 1984; Chan y Rubin, 1987; Lüder, 1992; Gandía y Archidona, 2008, Caba et al., 2008; Pina et al., 2010b). Por ello, se plantea como hipótesis que:

H2. Hay una asociación positiva entre los ingresos departamentales y la divulgación de información financiera $y$ presupuestal en los sitios web de los departamentos colombianos.

La teoría de la elección pública señala que los gerentes públicos que invierten de una manera más adecuada los re- 
cursos que gobiernan, son mejor valorados por los ciudadanos (Ball y Pflugrath, 2012). Al mismo tiempo se argumenta que para garantizar su reelección, los políticos realizan gastos de inversión que resulten más visibles para, y mejor valorados por, los votantes (Chan y Rubin, 1987; Cárcaba y García, 2008; Tamez, 2010). Por consiguiente se puede plantear la hipótesis según la cual:

H3. Hay una asociación positiva entre la magnitud de la inversión y la divulgación de información financiera y presupuestal en los sitios web de los departamentos colombianos.

Lüder (2002) señaló que un estímulo determinante de las reformas de la gestión pública es el estrés financiero. Banker y Patton, (1987), Zimmerman, (1977), Gandía y Archidona, (2008) y Caba et al., (2008), encontraron que cuando una entidad pública tiene un mayor endeudamiento, existen incentivos para realizar una divulgación voluntaria de información, para buscar disminuir el costo de la deuda. En este contexto, la capacidad para pagar las deudas, cubriendo los intereses y amortizando el principal, es una medida de la presión financiera de una entidad, más comprensiva que el costo de los intereses (Ball y Pflugrath, 2012; IFAC, 2012). Por consiguiente, los gerentes públicos tienen incentivos para mostrar la capacidad de las entidades que administran, para honrar la deuda en el futuro (IFAC, 2011). En consecuencia se plantean dos hipótesis:

H4. Hay una asociación positiva entre el estrés financiero (servicio de la deuda) y la divulgación de información fi- nanciera y presupuestal en los sitios web de los departamentos colombianos.

H5. Hay una asociación positiva entre el apalancamiento departamental y la divulgación de información financiera y presupuestal en los sitios web de los departamentos colombianos.

Para contrastar cada una de estas hipótesis se desarrollaron cuatro modelos de regresión lineal por MCO. Las variables dependientes de cada modelo son los índices específicos construidos y el Índice de Divulgación Global. Se han seleccionado siete variables independientes, de las cuales cinco (5) están directamente alineadas con cada hipótesis y dos (2) son variables de control. Las variables están definidas en el Cuadro 1.

Los modelos de regresión propuestos se pueden expresar como:

ICI $=\beta_{0}+\beta_{1}$ POBTOTAL $+\beta_{2^{*}}$ PIBDEPAR $+\beta_{3^{*}}$ PENINTER $+\beta_{4^{*}}$ INGTOTAL + $\beta_{5} *$ MAGINVER $+\beta_{6}$ SERVIDEUDA+ $\beta_{7}$ APALANC

(Modelo 1)

ICC $=\beta_{0}+\beta_{1} *$ POBTOTAL $+\beta_{2}$ PIBDEPAR $+\beta_{3^{*}}$ PENINTER $+\beta_{4^{*}}$ INGTOTAL + $\beta_{5^{*}}$ MAGINVER $+\beta_{6^{*}}$ SERVIDEUDA+ $\beta_{7^{*}}$ APALANC

(Modelo 2)

INA $=\beta_{0}+\beta_{1^{*}}$ POBTOTAL $+\beta_{2^{*}}$ PIBDEPAR $+\beta_{3^{*}}$ PENINTER $+\beta_{4} \pm$ INGTOTAL + $\beta_{5^{*}}$ MAGINVER $+\beta_{6^{*}}$ SERVIDEUDA+ $\beta_{7}$ APALANC

(Modelo 3)

IGD $=\beta_{0}+\beta_{1^{*}}$ POBTOTAL $+\beta_{2^{*}}$ PIBDEPAR $+\beta_{3^{*}}$ PENINTER $+\beta_{4^{*}}$ INGTOTAL + $\beta_{5^{*}}$ MAGINVER $+\beta_{6^{*}}$ SERVIDEUDA + $\beta_{7^{*}}$ APALANC

(Modelo 4)

Fue utilizada la prueba de Pearson para evaluar la correlación entre las variables independientes. Esta prueba es am- 


\section{Cuadro 1}

\section{Codificación, definición y fuentes de información de las variables independientes}

\begin{tabular}{|c|c|c|c|c|}
\hline Variable & Codificación & $\begin{array}{c}\text { Signo } \\
\text { esperado }\end{array}$ & Definición & Fuente \\
\hline $\begin{array}{l}\text { Población } \\
\text { (Variable } \\
\text { de control) }\end{array}$ & POBTOTAL & + & $\begin{array}{l}\text { Número de Habitantes } \\
\text { Logaritmo Neperiano Número de } \\
\text { habitantes. Año } 2010\end{array}$ & $\begin{array}{l}\text { Departamento Administrativo Na- } \\
\text { cional de Estadística -DANE. } \\
\text { www.dane.gov.co }\end{array}$ \\
\hline $\begin{array}{l}\text { Riqueza } \\
\text { (Variable } \\
\text { de Control) }\end{array}$ & PIBDEPAR & + & $\begin{array}{l}\text { Producto Interno Bruto del De- } \\
\text { partamento dividido por el Pro- } \\
\text { ducto Interno Bruto Nacional } \\
\text { Porcentaje. Año } 2010\end{array}$ & $\begin{array}{l}\text { Departamento Administrativo Na- } \\
\text { cional de Estadística -DANE. } \\
\underline{\text { www.dane.gov.co }}\end{array}$ \\
\hline $\begin{array}{l}\text { Acceso } \\
\text { a Internet }\end{array}$ & PENINTER & + & $\begin{array}{l}\text { Porcentaje de personas abonadas } \\
\text { (suscritas) a servicio de internet } \\
\text { en el hogar en el departamento, } \\
\text { en relación al total poblacional. } \\
\text { Porcentaje. Año } 2010\end{array}$ & $\begin{array}{l}\text { Ministerio de las Tecnologías de la } \\
\text { Información y las Comunicaciones. } \\
\text { Boletín trimestral TIC, cuarto tri- } \\
\text { mestre 2010. www.mintic.gov.co }\end{array}$ \\
\hline $\begin{array}{l}\text { Ingresos } \\
\text { Totales }\end{array}$ & INGTOTAL & + & $\begin{array}{l}\text { Cifra de ingresos totales } \\
\text { Sumatoria de ingresos corrientes } \\
\text { y los ingresos de capital (no in- } \\
\text { cluye financiamiento). } \\
\text { Logaritmo Neperiano del monto } \\
\text { de ingresos totales. Año } 2010\end{array}$ & $\begin{array}{l}\text { Departamento Nacional de Pla- } \\
\text { neación. www.dnp.gov.co } \\
\text { Sistema Consolidador de Hacien- } \\
\text { da e Información Financiera Pú- } \\
\text { blica - CHIP-.www.chip.gov.co }\end{array}$ \\
\hline $\begin{array}{l}\text { Magnitud de la } \\
\text { Inversión }\end{array}$ & MAGINVER & + & $\begin{array}{l}\text { Cifra del rubro gastos de inver- } \\
\text { sión, dividida por el monto total } \\
\text { de gastos del departamento. } \\
\text { Porcentaje. Año } 2010\end{array}$ & $\begin{array}{l}\text { Departamento Nacional de Pla- } \\
\text { neación. www.dnp.gov.co } \\
\text { Sistema Consolidador de Hacien- } \\
\text { da e Información Financiera Pú- } \\
\text { blica - CHIP-. www.chip.gov.co }\end{array}$ \\
\hline Estrés financiero & SERVIDEUDA & + & $\begin{array}{l}\text { Cifra que agrega los intereses de } \\
\text { la deuda pública y las amortiza- } \\
\text { ciones de la misma en el periodo. } \\
\text { Logaritmo Neperiano del monto } \\
\text { total de intereses y amortizacio- } \\
\text { nes de deuda. } \\
\text { Año } 2010\end{array}$ & $\begin{array}{l}\text { Departamento Nacional de Pla- } \\
\text { neación. www.dnp.gov.co } \\
\text { Contraloría General de la Repú- } \\
\text { blica. www.cgr.gov.co }\end{array}$ \\
\hline Apalancamiento & APALANC & + & $\begin{array}{l}\text { Monto total del saldo de la deuda } \\
\text { financiera dividido entre los in- } \\
\text { gresos totales. } \\
\text { Porcentaje. Año } 2010 \text {. }\end{array}$ & $\begin{array}{l}\text { Departamento Nacional de Pla- } \\
\text { neación. www.dnp.gov.co } \\
\text { Contraloría General de la Repú- } \\
\text { blica. www.cgr.gov.co }\end{array}$ \\
\hline
\end{tabular}

Fuente: Elaboración propia, proceso de definición metodológica de fuentes para construcción de variables explicativas, 2012. 
pliamente usada en la literatura sobre divulgación de información en Internet (Caba et al., 2008). Así mismo se ha realizado la estimación de los factores de inflación de la varianza FAV. La Tabla 5 resume la matriz de correlaciones de Pearson.

Se observa que existe una alta correlación entre las variables Población e Ingreso Total $(0,806)$, Riqueza departamental e Ingreso Total $(0,752)$, Riqueza departamental y Acceso a Internet $(0,805)$, y Población y Acceso a Internet $(0,707)$. Estas correlaciones son significativas con un P-valor 0,01 . Los factores de inflación de la varianza de estas variables no son extremos (Wooldridge, 2006).

Las variables Población y Riqueza son ampliamente usadas en la literatura como variables de control (Caba et al., 2008; Tamez, 2010). La variable Acceso a Internet ha sido contrastada en diferen- tes trabajos, en los que las variables de control también han sido las usadas en esta investigación (Gandía y Archidona, 2008; Caba et al., 2008). Los ingresos de un departamento dependen, en gran medida, del tamaño de la población; pero también se relacionan con factores tales como las transferencias del nivel central y de los recursos de participación en las regalías. Precisamente las regalías también hacen que los departamentos con poblaciones bajas, pero con alta concentración de recursos naturales no renovables $^{7}$, tengan participaciones en el PIB que no siempre siguen una relación lineal positiva entre población y riqueza. Por consiguiente, su correlación no implica co-linealidad en sentido estricto (Wooldridge, 2006).

Al retirar una de las dos variables de control, los resultados de significatividad de las variables explicativas conside-

\section{Tabla 5}

\section{Matriz de correlaciones de Pearson}

\begin{tabular}{|c|c|c|c|c|c|c|c|}
\hline & PobtotaL & Peninter & Pibdepar & Maginver & Servideuda & Ingtotal & Apalanc \\
\hline POBTOTAL & 1 & & & & & & \\
\hline PENINTER &, $707^{*}$ & 1 & & & & & \\
\hline PIBDEPAR & ,622 &, $805^{*}$ & 1 & & & & \\
\hline MAGINVER &,- 123 &,- 314 &,- 335 & 1 & & & \\
\hline SERVIDEUDA &, 576 &, 547 & ,469 &,- 248 & 1 & & \\
\hline INGTOTAL &, $806^{*}$ & ,696 &, $752^{*}$ &,- 052 & ,656 & 1 & \\
\hline $\begin{array}{l}\text { APALANC } \\
n=32 \cdot P, 01^{*}\end{array}$ & ,414 & ,447 & ,448 &,- 601 & ,617 & ,482 & 1 \\
\hline
\end{tabular}

Fuente: Elaboración propia a partir de SPSS, 2012. en 2010. 
radas individualmente no varían sustancialmente, pero se modifica el R-cuadrado ajustado del modelo. Lo anterior significa que las variables aportan de manera separada a la explicación de los modelos. Por consiguiente la eliminación de una de estas variables no resulta conveniente. En consecuencia, tal y como lo sugiere Wooldridge (2006), se decidió mantener las variables de control correlacionadas, ya que estas no son el foco de explicación de los modelos. La bondad de ajuste $\left(R^{2}\right)$ en los modelos 1,2 y 4 , resulta elevada, lo que se atribuye a que las variables seleccionadas han sido significativas en múltiples trabajos previos y aquí se usan de manera conjunta (Laswad et al., 2005; Martínez, 2005).

Los resultados de las regresiones son presentados en las Tablas 6. y 7 .

Como se observa, en todos los modelos la relación que tiene la variable Ingresos totales (INGTOTAL) con cada uno de los índices específicos y con el Índice Global de Divulgación es negativa. Por consiguiente, en el caso de los departamentos colombianos, en el periodo estudiado, no se identifica una asociación positiva entre los ingresos y la DIFI, por lo que es rechazada la hipótesis 2 .

De igual manera, la Magnitud de la Inversión (MAGINVER) en tres de los cuatro modelos, tiene una relación negativa con los índices. En cuanto a su relación con el índice de contenido de la información, pese a ser positiva, no resulta significativa pues su P-valor es de 0,894. En consecuencia, se rechaza la hipótesis No 3, puesto que no se evidencia una relación positiva entre la magnitud de la inversión y la DIFI.

Por su parte, el Acceso a Internet (PENINTER) tiene una relación positiva con todos los índices específicos y con el Índice Global de Divulgación. Su impacto en el modelo del Índice Global y en el del Índice de Características Cualitativas es significativa con un $\mathrm{P}$-valor $\mathrm{0}, 05$, mientras que en el modelo del Índice de Contenido de la Información, su significatividad es un poco más moderada puesto que el $\mathrm{P}$ valor es 0,1 . En síntesis, se puede decir que en el caso colombiano, existe una relación positiva entre el acceso a Internet en los departamentos y la DIFI departamental.

En el mismo sentido, la variable Apalancamiento (APALANC) muestra una relación positiva en todos los modelos. El impacto de esta variable en el Índice de Contenido y en el Índice Global de Divulgación es significativa con un P-valor 0,10 . No obstante, la significatividad de esta variable para explicar el modelo del índice de contenido es baja, puesto que su $p$-valor es de 0,136. A pesar de esto se identifica una relación positiva entre el apalancamiento y la DIFI departamental en Colombia, por lo que no es posible rechazar la hipótesis 5 .

Por su parte, la variable Estrés Financiero (SERVIDEUDA) sólo presenta una relación negativa con el Índice de $\mathrm{Na}$ vegabilidad y Acceso, mientras que es positiva con los otros índices. Como se ha evidenciado con los resultados presentados y se discutirá en el siguiente acápite, la navegabilidad y el acceso mayoritariamente muestran relaciones negativas con las variables explicativas. En cuanto al índice de Contenido y al Índice Global de Divulgación la relación de esta variable es significativa con un P-valor de 0,05 y de 0,067 respectivamente. En consecuencia, no es posible rechazar la hipótesis 4 , 


\section{Tabla 6}

Resultado de las regresiones por MCO, Modelos 1 y 2

\begin{tabular}{|c|c|c|c|c|c|c|c|c|}
\hline & \multicolumn{4}{|c|}{$($ Modelo 1) - ICI - } & \multicolumn{4}{|c|}{ (Modelo 2) - ICC - } \\
\hline & $\begin{array}{l}\text { Exp. } \\
\text { Sign }\end{array}$ & $\begin{array}{c}\text { Standardized } \\
\text { Coefficients }\end{array}$ & $\mathrm{T}$ & Sig. & $\begin{array}{l}\text { Exp. } \\
\text { sign }\end{array}$ & $\begin{array}{c}\text { Standardized } \\
\text { Coefficients }\end{array}$ & $\mathrm{T}$ & Sig. \\
\hline & \multicolumn{3}{|c|}{ Beta } & \multicolumn{5}{|c|}{ Beta } \\
\hline Pobtotal & + &, $631^{* *}$ & 2,279 & ,032 & + &, $696^{\star *}$ & 2,242 & ,035 \\
\hline Peninter & + &, $338^{*}$ & 1,832 &, 079 & + &, $490^{\star *}$ & 2,365 & ,026 \\
\hline Pibdepar & + & 051 & ,223 & ,826 & + &,- 065 &,- 252 & ,803 \\
\hline Maginver & + & ,020 & ,135 & ,894 & + &,- 067 &,- 400 & ,693 \\
\hline Servideuda & + &, $428^{* * *}$ & 3,094 &, 005 & + &, $302^{*}$ & 1,945 & ,064 \\
\hline Ingtotal & + &,- 622 & $-1,657$ & ,110 & + &,$- 734^{*}$ & $-1,743$ & ,094 \\
\hline Apalanc & + &, $270^{*}$ & 1,771 & ,089 & + & ,264 & 1,543 & ,136 \\
\hline \multicolumn{5}{|c|}{$n=32 / R^{2}=0,762 ;{ }^{*} p<0,10 ;{ }^{* *} p<0,05 ;{ }^{* * *} p<0,01$} & \multicolumn{4}{|c|}{$\mathrm{R}^{2}=0,701{ }^{*} p<0,10 ;{ }^{* *} p<0,05 ;{ }^{* * *} p<0,01$} \\
\hline
\end{tabular}

Fuente: Elaboración a partir de regresiones, SPSS, 2012.

\section{Tabla 7}

Resultado de las regresiones por MCO, Modelos 3 y 4

\begin{tabular}{|c|c|c|c|c|c|c|c|c|}
\hline & \multicolumn{4}{|c|}{ (Modelo 3) -INA- } & \multicolumn{4}{|c|}{ (Modelo 4) -IGD- } \\
\hline & $\begin{array}{l}\text { Exp. } \\
\text { Sign }\end{array}$ & $\begin{array}{c}\text { Standardized } \\
\text { Coefficients }\end{array}$ & $\mathbf{T}$ & Sig. & $\begin{array}{l}\text { Exp. } \\
\text { sign }\end{array}$ & $\begin{array}{c}\text { Standardized } \\
\text { Coefficients }\end{array}$ & $\mathrm{T}$ & Sig. \\
\hline & \multicolumn{4}{|c|}{ Beta } & \multicolumn{4}{|c|}{ Beta } \\
\hline Pobtotal & + & ,367 & ,828 & ,416 & + &, $649^{* *}$ & 2,185 & ,039 \\
\hline Peninter & + & ,297 & 1,006 & ,325 & + &, $429^{* *}$ & 2,165 & 041 \\
\hline Pibdepar & + &,- 150 &,- 410 & ,686 & + &,- 053 &,- 215 & ,831 \\
\hline Maginver & + &,$- 416^{\star}$ & $-1,734$ & ,096 & + &,- 141 &,- 876 & ,390 \\
\hline Servideuda & + &,- 021 &,- 095 & ,925 & + &, $287^{*}$ & 1,935 & 065 \\
\hline Ingtotal & + &,- 458 &,- 762 & ,454 & + &,$- 689^{*}$ & $-1,709$ & 100 \\
\hline Apalanc & + & ,296 & 1,217 & 236 & + & ,302 & 1,848 & ,077 \\
\hline \multicolumn{5}{|c|}{$\mathrm{n}=32 / \mathrm{R}^{2}=0,391 ;{ }^{*} p<0,10 ;{ }^{* *} p<0,05 ;{ }^{* *} p<0,01$} & \multicolumn{4}{|c|}{$\mathrm{R}^{2}=0,726 ;{ }^{*} p<0,10 ;{ }^{* *} p<0,05 ;{ }^{* * *} p<0,01$} \\
\hline
\end{tabular}

Fuente: Elaboración a partir de regresiones, SPSS, 2012. 
puesto que el Estrés Financiero se relaciona positivamente con el contenido de la información divulgada y con el Índice Global de Divulgación.

Finalmente, en cuanto a las variables de control seleccionadas, en el caso de este trabajo la Riqueza departamental (PIBDEPAR) muestra una relación positiva con todos los índices, pero su participación no es significativa como lo manifiestan los P-valores. Al mismo tiempo, se verifica que la Población (POBTOTAL) tiene una relación positiva y altamente significativa con la DIFI (P-valor,05) en todos los modelos.

En comparación con los resultados obtenidos para los gobiernos centrales en la región latinoamericana (Rodríguez et al., 2006), los departamentos colombianos presentan un nivel muchísimo más bajo de DIFI ( $34,80 \%$ versus $82,5 \%$ ). Esto puede deberse a que los gobiernos centrales se encuentran más expuestos al control parlamentario y a las exigencias de organizaciones multilaterales (Godfrey, et al., 1996). Es necesaria más investigación para identificar la relación entre la descentralización administrativa y el nivel de DIFI en el país, lo que abre puertas a la investigación futura.

Dado que 14 departamentos tienen niveles de divulgación por debajo del $20 \%$, se evidencian desequilibrios en la implantación y en los logros de Gobierno en Línea a nivel territorial. A la vez, dado que los departamentos con los peores puntajes en el Índice General de Divulgación-IGD-son las antiguas "intendencias y comisarías", se pone de manifiesto que el grado de desarrollo regional impacta la implantación del gobierno electrónico
(Rodríguez, 2006), particularmente en materia de DIFI.

Los puntajes del Índice de Navegabilidad y Acceso -INA-, así como los resultados de la regresión del modelo 3 revelan tendencias disímiles, en relación con los otros modelos. Esto se debe a que la estrategia de Gobierno en Línea obliga la creación de un sitio web, a partir de un formato predefinido. En conclusión, los resultados en INA no se explican por variables financieras o socioeconómicas, sino por el cumplimiento de la obligación legal. Con ello prevale el mandato de la ley (rule of law), como ha sido evidenciado en otros contextos con tradiciones de derecho romano (Pina et al., 2010b; Guillamón et al., 2011).

El tipo de información que más se divulga es presupuestal, lo que evidencia su prevalencia en la cultura administrativa del sector público para la toma de decisiones y la rendición de cuentas (Benito et al., 2007). Otro elemento asociado a ello, es la muy baja publicación en la web de los informes contables básicos, que deben prepararse y remitirse trimestralmente de manera electrónica a la Contaduría General de la Nación. Por lo tanto, publicar esta información no supondría un costo adicional. El Balance General constituye el informe financiero más divulgado, pero sólo 13 de los departamentos lo han dispuesto en la red. La presencia de indicadores sobre el cumplimiento de metas o eficacia, es también motivada por la exigencia de producir un informe de gestión anual para la audiencia pública de rendición de cuentas de las entidades (Departamento Administrativo de la Función Pública, 2005). 
El nivel de calidad de la información divulgada por los departamentos es del $29,04 \%$. Las diferencias en la observancia de las características cualitativas de la información siguen la tendencia de concentración en los departamentos con los mejores índices de contenido.

En cuanto a las variables explicativas de los niveles de divulgación, se verifica que el acceso a Internet tiene una relación positiva y significativa con la divulgación de información (Debreceny et al., 2002; Gandía y Archidona, 2008; Caba, et al., 2008). En los departamentos en los cuales la penetración de Internet en los hogares es mayor, hay más divulgación de información pública por este canal.

El apalancamiento es una variable que se verifica como significativa en la DIFI de los departamentos. El endeudamiento de los departamentos colombianos se incrementó después de la Constitución de 1991 ya que profundizó la descentralización durante la década de 1990 (Clavijo, 2011). Los departamentos con mayor tamaño poblacional se endeudaron, en parte, para cubrir las necesidades derivadas del traslado de competencias y por la necesidad de acometer inversiones para la prestación de servicios públicos bajo su responsabilidad (Sandoval, Gutiérrez y Guzmán, 2000).

Como consecuencia del endeudamiento creciente, muchos departamentos vivieron crisis de deuda y restructuraron sus obligaciones con los acreedores a inicios del 2000 (Gómez y García, 2007). Dado que el servicio de la deuda es una medida que recoge tanto el costo financiero como la amortización del principal, esta variable es un mejor indicador de la presión financiera a la que están expuestas las administraciones endeudadas (Lüder, 2002; IFAC, 2011). Se observa que los departamentos con mayor endeudamiento y mayor servicio de la deuda en 2010 , tienen unos mejores índices de divulgación de la información contable y presupuestal en Internet. Por consiguiente, el estrés fiscal puede constituirse en un promotor de reformas e innovaciones de la administración pública tales como el gobierno electrónico, con lo cual este trabajo contribuye a la literatura con evidencia empírica sobre la relevancia de esta variable.

En síntesis, las variables económico-financieras, sociales y demográficas que se relacionan con la divulgación de información de los departamentos son: el acceso a Internet, el apalancamiento y el estrés financiero. El tamaño de la población es también una variable de control que resulta significativa en el caso colombiano. Por el contrario los ingresos del departamento, la magnitud de la inversión y la riqueza departamental no resultan positiva ni significativamente relacionadas con los niveles de divulgación de información financiera y presupuestal en Internet.

\section{Conclusiones}

El gobierno electrónico se constituye en un medio de modernización de la administración pública que busca, entre otros objetivos, impulsar la transparencia y la rendición de cuentas. Algunas evidencias muestran que pese a los esfuerzos y recursos comprometidos en los proyectos de e-government, aún los resultados en tales propósitos son muy limitados. Lo anterior puede implicar que las expectativas probablemente han sido so- 
bredimensionadas, o quizás que el cambio y direccionamiento del sector público hacia la transparencia reclama transformaciones más allá de las innovaciones tecnológicas y simplemente comunicacionales.

En el caso de esta investigación se ha evaluado la divulgación y la transparencia de la información financiera y presupuestal en Internet (DIFI) de los departamentos colombianos. La estrategia nacional conocida como Gobierno en Línea, promueve la utilización de Internet para mejorar la interacción de la administración pública, en todos sus niveles, con la ciudadanía. Evaluar el avance y los logros de estas iniciativas en las entidades territoriales, permite apreciar con mayor detalle la profundidad de los cambios y la transformación real del sector público. El gobierno nacional implementa y asimila las reformas y las innovaciones con un mayor ritmo, pero los procesos en las regiones están condicionados por una marcada limitación de recursos humanos y financieros, procesos de desarrollo asimétrico y dinámicas socio-políticas de alta complejidad.

La tradición centralista del Estado en Latinoamérica, ha implicado que las políticas públicas y las reformas se planteen a la medida de las condiciones de los gobiernos centrales, antes que atendiendo a las necesidades de los ciudadanos en las regiones y territorios apartados. Esto parece ocurrir con la implementación del e-government en los departamentos de Colombia, lo que se manifiesta en los bajos niveles de divulgación de información que tales entidades realizan por medio de Internet, y que evidencia un particular rezago en las entidades más alejadas de los centros de poder político y económico.
No obstante lo anterior, también se puede señalar que la divulgación de información pública por medio de Internet está obligando a las entidades a una mayor apertura informativa. Aunque los niveles de transparencia son bajos, la ciudadanía comienza a reconocer en las TIC's un potente instrumento de información y conocimiento de las acciones públicas. Los políticos y gerentes públicos comienzan a incorporar en sus agendas la gestión de la información pública por medios electrónicos. Pese a ello, aún está por identificarse si la información que se viene disponiendo en Internet, particularmente la financiera y presupuestal, resulta comprensible y útil para los ciudadanos.

Los retos contemporáneos en materia de e-government y transparencia pasan por identificar las barreras reales al acceso, a la interpretación y al uso de la información pública. Quizás más información no es suficiente, sino incluso en ocasiones contraproducente. Se requiere información relevante, pertinente y comprensible, antes que un gran volumen de datos que los ciudadanos no pueden interpretar y que, en apariencia, dan la imagen de apertura de las entidades y sus gestores.

\section{Referencias bibliográficas}

Anderson, James y Zahaf, Mehdi (2007). Profiling consumer to consumer and Business to Consumer Buyers: Who buys what? International Journal of Business Research, Vol. 7, No 6, pp 85-99.

Asamblea Nacional Constituyente (1991). Constitución Política de Colombia. Gaceta del Congreso. Bogotá. Colombia.

Asociación Española de Contabilidad y Administración de Empresas, AECA. 
(2005). Código de buenas prácticas para la divulgación de información en Internet. Documento 1-serie Nuevas tecnologías y contabilidad. Asociación Española de Contabilidad y Administración. Madrid.

Ball, lan y Pflugrath, Gary (2012). Government Accounting. Making Enron look good. World Economics, 13 (1), pp 9-26.

Banker, Rajiv.D. y Patton, James (1987). Analytical agency theory and municipal accounting: an introduction and an application. Research in Governmental and Nonprofit Accounting, Vol. 3, pp. 29-50.

Benito, Bernardino, Brusca, Isabel y Montesinos, Vicente (2007). The Harmonization of Government Financial Information Systems: the Role of the IPSASs. International Review of Administrative Sciences, Vol. 73, No. 2, pp. 293-317.

Caba Carmen, López, Antonio Manuel y Rodríguez, Manuel (2005). Citizens' access to on-line governmental financial information: practices in the European Union countries. Government Information Quarterly, Vol. 22 No. 2, pp. 258-76.

Caba Carmen, Rodríguez, Manuel y López, Antonio Manuel (2008). E-Government process and incentives for online public financial information. Online Information Review Vol. 32 No. 3, pp. 379-400.

Cárcaba, Ana y García, Jesús (2008). Determinantes de la divulgación de información contable a través de Internet por parte de los gobiernos locales. Revista Española de Financiación y Contabilidad, Vol. XXXVII, No. 137, enero-marzo 2008, pp. 63-84

Chan, James y Rubin, Marc (1987). The Role of Information in a Democracy and in Government Operations: The Public Choice Methodology. Research in
Governmental and Nonprofit Accounting, Volume 3, Part B, pp. 3-27.

Christensen, Mark y Skaerbaek, Peter (2010). Consultancy outputs and the purification of accounting technologies. Accounting, Organizations and Society, 35, 524-545.

Clavijo, Sergio (2011). Estructura Fiscal de Colombia y Ajustes Requeridos 2010-2020. Premio a la Investigación "Germán Botero de los Ríos"-Fedesarrollo. Asociación Nacional de instituciones Financieras-ANIIF. Bogotá.

Collin, Sven-olf, Tagesson, Torbjörn, Andersson, Anette, Cato, Joosefin, y Hansson, Karin. (2009), Explaining the choice of accounting standards in municipal corporations: Positive accounting theory and institutional theory as competitive or concurrent theories. Critical Perspectives on Accounting 20, pp 141-174.

Congreso de la República de Colombia (2005). Ley 962 de 2005. Por el que se establecen medidas de racionalización de trámites. Gaceta del Congreso. Bogotá. Colombia.

Contaduría General de la Nación (2007). Resolución 354/2007 de la Contaduría General de la Nación. Por la cual se adopta el Régimen de Contabilidad Pública. Diario Oficial. Bogotá. Colombia.

Contraloría General de la República. Base de Datos de Estadísticas de las Finanzas Públicas. www.cgr.gov.co. Consultado el 20 de Junio de 2012.

Culebro, Jorge (2008). Transferencia de Políticas y construcción de lecciones para la modernización administrativa. Revista Venezolana de Gerencia, 13 (44), pp 531-542.

D’Agostino, Maria, Schwester, Richard, Carrizales, Tony, y Melitski, James (2011). A Study of E-government and E-go- 
vernance: An Empirical Examination of Municipal Websites. Public Administration Quarterly, 35, 1, pp 3-26.

Debreceny, Roger, Gray, Glen, y Rahman, Asheq (2002). The determinants of Internet financial reporting, Journal of Accounting and Public Policy 21 (4-5), 371-394.

Departamento Administrativo de la Función Pública, DAFP (2005). Guía para la Rendición de cuentas de la Administración Pública a la Ciudadanía. Bogotá. Colombia.

Departamento Administrativo Nacional de Estadística-DANE. Base de datos censo poblacional. www.dane.gov.co. Consultada el 20 de Mayo de 2012.

Departamento Nacional de Planeación-DNP. Base de datos de las Finanzas Públicas Territoriales. www.dnp.gov.co. Consultado el 26 de Julio de 2012.

Di Maggio, Paul, y Powell, Walter (1983). The Iron Cage Revisited: Institutional Isomorphism and Collective Rationality in Organizational Fields. American Sociological Review, Vol. 48 pp.147 - 160.

Financial Accounting Standards Board, FASB (2000). Electronic Distribution of Business Reporting information. Steering Committee Report Series. Norwalk. United States of America.

Gandía, Juan Luís y Archidona, María (2008), Determinants of web site information by Spanish city councils. Online Information Review, 32, 1, pp. 35-57.

Godfrey, Alan, Devlin, Patrick y Merrouche, Cherif (1996), Governmental Accounting in Kenya, Tanzania and Uganda. Research in Governmental and Nonprofit Accounting, Vol 9. pp 193-208.

Gómez, Bersarión. y García, Lilibeth (2007), La deuda externa y territorial en Colombia: Evolución, Impacto y Análisis de la situación Actual. Diálogos de saberes (26), pp. 117-146.
Guillamón, María, Bastida, Francisco and Benito, Bernardino (2011). The Determinants of Local Government's Financial Transparency. Local Governent Studies, Vol. 37, (4), 391-406.

Ho, Alfred (2002). Reinventing Local Governments and the E-Government Initiative, Public Administration Review, Vol. 62, No. 4, pp. 434-44.

Hood, Christopher (1995). The "New Public Management" in the 1980s: Variations on a Theme. Accounting, Organizations and Society, 20, 2/3, pp 931009.

Hood, Christopher (2010). Accountability and Transparency: Siamese Twins, Matching Parts, Awkward Couple? West European Politics, Vol. 33, No. 5, 989-1009.

Ingram, Robert (1984). Economic Incentives and the Choice of State Government Accounting Practices. Journal of Accounting Research, 22 (1), 126144.

International Accounting Standards Committee, IASC (1999). Business Reporting on Internet. International Accounting Standards Committee discussion paper. London.

International Federation of Accountants, IFAC. (2011). Reporting on the LongTerm Sustainability of a Public Sector Entity's Finances. Proposed Recommended Practice Guideline. Exposure Draft 46. Toronto.

International Federation of Accountants, IFAC (2012). Public Sector Financial Management Transparency and Accountability: The use of International Public Sector Accounting Standards. Policy Position Paper No 4. IPSASB. March. New York.

Items International y Moreno, Hernán (2007). E-government architectures, technical and political situation in Latin America. Proyect Documents co- 
llection N 19. Comisión Económica para América Latina y el Caribe-CEPAL y Oficina de cooperación para la Ayuda Europea. Santiago de Chile.

King, Julie (2006). Democracy in the information age. Australian Journal of Public Administration, Vol 65, No 2. pp 16-32.

La Porte, Todd, Demchak, Chris y De Jong, Martin (2002). "Democracy and bureaucracy in the age of the web. Empirical findings and theoretical speculations", Administration and Society, Vol. 34 No. 4, pp. 411-446.

Lapsley, Irvine, y Miller, Peter (2010). The Egovernment proyect. Financial Accountability and Management, $26,1, \mathrm{pp} 1-2$.

Lapsley, Irvine (2009). New Public Management: The Cruellest Invention of Human Spirit? Abacus, 45, 1, pp 1-21.

Laswad, Fawzi, Fisher, Richard, y Oyelere, Peter (2005). "Determinants of voluntary Internet financial reporting by local government authorities", Journal of Accounting and Public Policy, Vol. 24, pp. 101-21.

Lüder, Klaus (1992). A contingency model of governmental accounting innovations, in the political-administrative environment. Research in Governmental and Nonprofit Accounting, 7, 99-127.

Lüder, Klaus (2002). Research in Comparative Governmental Accounting over the last decade-Achievements and Problems-. En: V. Montesinos y J.M. Vela (Eds) Innovations in Governmental Accounting. Kluwer Academic Publishers.

Martínez, Elena (2005). Errores frecuentes en la interpretación del coeficiente de determinación lineal. Anuario Jurídico y Económico Escurialense, XXXVIII, pp 315-332.

Ministerio de Comunicaciones (2008). Decreto 1151 de 2008. Por el cual se esta- blecen los lineamientos generales de la Estrategia de Gobierno en Línea de la República de Colombia. Diario Oficial. Bogotá. Colombia.

Ministerio de Hacienda y Crédito Público (1996). Decreto 111 de 1996. Por el cual se establece el Estatuto Orgánico del Presupuesto. Diario Oficial. Bogotá. Colombia.

Ministerio de las Tecnologías de la Información y las Comunicaciones. (2010). Boletín trimestral TIC, cuarto trimestre. www.mintic.gov.co. Consultado el 10 de mayo de 2012.

Ministerio de las Tecnologías de la Información y las Comunicaciones, MTIC. (2011). El gobierno en línea en Colombia 2010-2011. Programa Gobierno en Línea. Bogotá. Colombia.

Montesinos, Vicente (2009). Transparencia y responsabilidad en el sector público: el papel de la información en tiempos de crisis. Revista AECA N.87, especial XV congreso, 26-28.

Moon, Jae (2002). '¿The Evolution of E-Government Among Municipalities: Rhetoric or Reality?' Public Administration Review, Vol. 62, No. 4, pp. 42433.

National Audit Office (2002). Better Public Service through E-government. Stationery Office. London. United Kingdom.

Organisation for Economic Co-operation and Development, OECD (2003). The Egovernment imperative. OECD eGovernment Studies. París. France.

Pilcher, Robyn (2011). Implementing IFRS in Local Government: Institutional Isomorphism as NPM Goes Mad? Local Government Studies, 37, 4, 367-389.

Pina, Vicente, Torres, Lourdes, and Royo, Aana (2010b). Is e-government promoting convergence towards more accountable local governments? International Public Management Journal, 13,4 pp 350-380. 
Pina, Vicente, Torres, Lourdes, and Royo, Ana (2010a). ¿ls e-government leading to more accountable and transparent local governments? An overall view. Financial Accountability and Management, 26, (1), pp 3-20.

Rodríguez, Manuel, Caba, Carmen, López, Antonio (2006). Cultural contexts and governmental digital reporting. International Review of Administrative Sciences, 72, (2), pp 269-290.

Rodríguez, Martha (2006). Investigación sobre el estado de avance del gobierno electrónico en Colombia a nivel municipal, regional y nacional. Cátedra Software AG-Alianza Sumaq en e-Government. Facultad de Administración. Universidad de los Andes. Bogotá. Colombia

Sandoval, Carlos, Gutiérrez, Javier y Guzmán, Carolina (2000), Colombia y la deuda pública territorial. Estudios de Economía y Ciudad No. 8. Secretaria de Hacienda Distrital. Bogotá D.C.http://impuestos.shd.gov.co/portal/page/portal/portal_Internet_sdh/publicaciones/Est_fis_pub/econoyciudad_est_pub/ESTUDIO08_DEE.pdf. Extraído el 20 de Mayo de 2012.

Serrano, Carlos, Rueda, Mar and Portillo, Pilar (2009), Determinants of e-government extension. Online Information Review Vol. 33 No. 3, pp. 476-498.

Sistema Consolidador de Hacienda e Información Pública -CHIP-. www.chip. gov.co. Consultado el 17 de Julio de 2012.

Sour, Laura (2007). Evaluando el gobierno electrónico: avances en las finanzas públicas estatales. Economía, So- ciedad y Territorio, Vol. VI, No 23. pp 613-654

Tamez, Silverio (2010). Rendición de cuentas, divulgación y control de la información de los gobiernos estatales en México. Tesis Doctoral. Facultad de Ciencias Económicas y Empresariales. Universidad de Cantabria. Santander. España.

Transparencia por Colombia (2009). Índice de Transparencia Departamental 20082009. Informe de la corporación Transparencia por Colombia. Bogotá. Colombia.

United Nations (2010). E-Government survey 2010. Leveraging e-government at a time of financial and economic crisis. United Nations. New York. United States of America.

United Nations (2012). E-Government survey 2010. E-government for people. United Nations. New York. United States of America.

Vargas, Carlos (2010). Desarrollo y E-gobierno en Latinoamérica: Responsabilidad y Transparencia de la información Económico-financiera de la gestión pública. Tesis Doctoral. Universidad de Granada. Granada. España.

West, Darrell (2004). E-Government and the Transformation of Service Delivery and Citizen Attitude. Public Administration Review, Vol. 64, No. 1, pp. 15-27.

Wooldridge, Jeffrey (2006). Introducción a la econometría. 2 edición. Thomson. Barcelona. España.

Zimmerman, Jerold (1977). The municipal accounting maze: an analysis of political incentives, Journal of Accounting Research, Vol. 15, Suppl., pp. 107-144. 